\title{
Quantifying the Matrix
} Metalloproteinase 2 (MMP2) Spatially in Tissues by Probe via MALDI Imaging Mass Spectrometry

\author{
Daojiang Yu ${ }^{1 * t}$, Peng Lai ${ }^{2+}$, Tao Yan ${ }^{1}$, Kai Fang ${ }^{1}$, Lei Chen ${ }^{1}$ and Shuyu Zhang ${ }^{1,3 *}$ \\ ${ }^{1}$ The Second Affiliated Hospital of Chengdu Medical College, China National Nuclear Corporation 416 Hospital, Chengdu, China, \\ ${ }^{2}$ Department of Endocrinology, Xuzhou Center Hospital, Xuzhou, China, ${ }^{3}$ Department of Oncology, The Affiliated Changzhou No. \\ 2 People's Hospital of Nanjing Medical University, Changzhou, China
}

\section{OPEN ACCESS}

Edited by:

Cheng Guo,

Zhejiang University, China

Reviewed by: Samuel M Meier-Menches, University of Vienna, Austria Fei Fang,

Michigan State University, United States Jie Tan,

Hunan University, China

*Correspondence: Daojiang Yu ydj51087@163.com Shuyu Zhang zhang.shuyu@hotmail.com

${ }^{t}$ These authors have contributed equally to this work

Specialty section:

This article was submitted to Analytical Chemistry, a section of the journal Frontiers in Chemistry

Received: 30 September 2021 Accepted: 25 November 2021 Published: 15 December 2021

Citation: Yu D, Lai P, Yan T, Fang K, Chen L and Zhang S (2021) Quantifying the Matrix Metalloproteinase 2 (MMP2) Spatially in Tissues by Probe via MALDI Imaging

Mass Spectrometry.

Front. Chem. 9:786283.

doi: 10.3389/fchem.2021.786283
As a matrix metalloproteinase, the abnormal expression of MMP2 is associated with multiple biological processes, including tissue remodeling and cancer progression. Therefore, spatial analysis of MMP2 protein in tissues can be used as an important approach to evaluate the expression distribution of MMP2 in complex tissue environments, which will help the diagnosis and treatment of various diseases, including tissue or organ injuries. Moreover, this analysis will also help the evaluation of prognoses. However, MMP2 is difficult to be spatially determined by MALDI TOF mass spectrometry due to its large molecular weight (over $72 \mathrm{KD}$ ) and low content. Therefore, a new method should be developed to help this detection. Here, we have designed a specific MMP2 probe that closely binds to MMP2 protein in tissue. This probe has a $\mathrm{Cl}$ on Tyr at the terminal, which can provide two isotope peaks to help the accuracy quantitative of MMP2 protein. Based on this, we used the probe to determine the spatial expression of MMP2 in the tissues based on MALDI TOF mass spectrometry. This approach may help to study the influence of multifunctional proteases on the degree of malignancy in vivo.

Keywords: MMP2, MALDI-TOF/MS, spatial quantitative protein detection, tumor, tissue remodeling

\section{INTRODUCTION}

Matrix metalloproteinases (MMPs) are a superfamily of zinc-containing endopeptidases. MMPs play vital roles in the degradation of extracellular matrix components. They are also involved in various types of physical processes such as cell proliferation, migration, differentiation, apoptosis, and angiogenesis by interacting with certain cytokines and chemokine (Griselda et al., 2020). These processes occur to promote tissue or organ regeneration through actively remodeling. Moreover, the abnormal expression of MMPs would lead to serious diseases. For example, MMP2 can regulate tissue remodeling, a normal process in which old bone is broken down and new bone is created to replace it. At least eight mutations in the MMP2 gene have been documented to cause multicentric osteolysis, nodulosis, and arthropathy (MONA). In addition, MMP2 was also reported to be over expressed in several solid tumors, including gastric carcinoma, breast carcinoma, lung cancer, etc (Montalban-Arques and Scharl, 2019, Weng et al., 2019, Zhang D et al., 2019). Hence, it is of great importance to locate the expression MMP2 in complex tissues. 
GPLGVRGY-CI
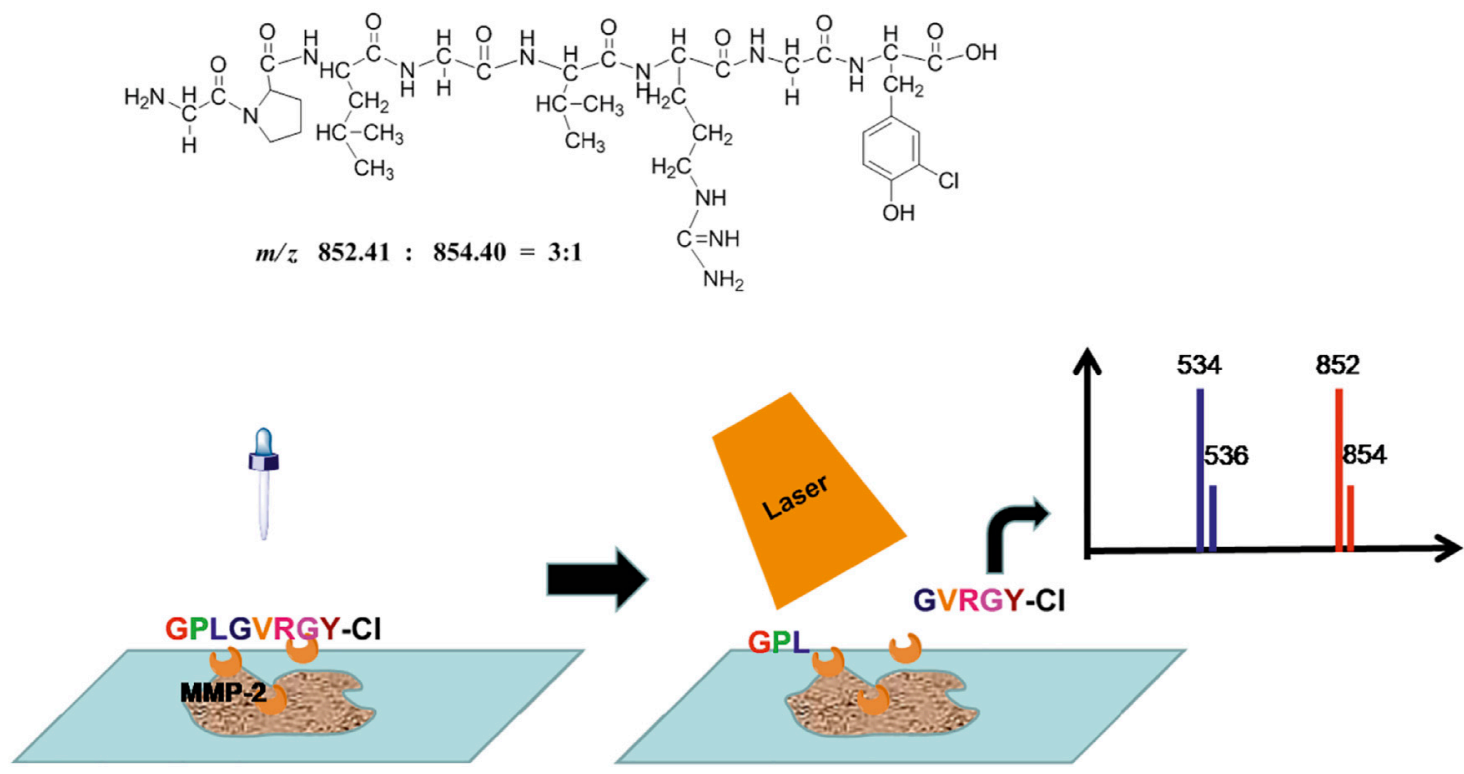

FIGURE 1 | The scheme of targeted spatial protein quantification strategy.

Matrix-assisted laser desorption/ionization mass spectrometry (MALDI MS) has become a powerful means of detection technology, which achieved more and more attention by clinical researchers (Barre et al., 2019; Balluff et al., 2021). Based on this method, even the spatial location of biomarkers in the organization can be provided by means of visualization (Shariatgorji et al., 2019). At present, this technique has successfully achieved the spatial analysis of small molecules, such as amino acids, lipids, nucleic bases, and partial macro-molecules, containing peptides and proteins (Moore et al., 2019; Boskamp and Soltwisch, 2020). However, it also has difficulty in the detection of proteins due to their large molecular weight and low content (Ryan et al., 2019). Although the MALDI technique is suitable for the detection of macromolecules, its sensitivity and resolution would decrease significantly when detecting proteins with a molecular weight greater than $20 \mathrm{KDa}$ (Zhang et al., 2019b). The MMP2 protein has a molecular weight of $72 \mathrm{kDa}$, which would provide challenges for MALDI spatial detection.

Herein, we have developed a strategy for the MMP2 MALDI spatial protein quantification determination. A peptide probe was designed containing the MMP2 digestion sequence. This peptide also had a chlorine atom on Tyr at the peptide terminal, which could provide two isotopes to increase detection accuracy. This peptide probe can be digested by MMP2 into a fragment containing isotope, and the content of MMP2 can be obtained according to the proportion between fragment and original probe. Additionally, this method has been successfully applied to the MMP2 imaging detection in CRC tissues, which can provide help for the prognoses and clinical medication of patients.

\section{MATERIALS AND METHODS}

The peptide probe was synthesized by BANKPEPTIDE Biological Technology CO (Anhui, China), with the $\mathrm{Cl}$ in the benzene ring of tyrosine at the $\mathrm{C}$ terminal. O-carboxy cinnamic acid (CHCA) was used as the matrix for MALDI analysis and purchased from Merck (United States). Acetonitrile $(\mathrm{ACN})$ was analytically pure and purchased from Sinopharm Technology CO (Shanghai, China).

A total of 20 couples of human cancer and tumor-adjacent tissues were collected from CRC patients in the form of frozen sections. These tissues are slices left from clinical biopsies. All samples were obtained with informed consent under a protocol approved by the Second Affiliated Hospital of Chengdu Medical College (No. E2021015). Patients' clinical information and pathological results were also recorded.

All tissues were sectioned at $10 \mu \mathrm{m}$ thickness and -20 C using a Leica cryostat (Leica Microsystems, Wetzlar Germany) and traw mounted onto indium tin oxide (ITO)- coated glass slides (4-8 $\Omega$ resistance, Delta Technologies, United States) and stored at $-80^{\circ} \mathrm{C}$ until analyzed.

For tissue targeted spatial protein quantification experiments, three layers of peptide prove $(0.5 \mathrm{mg} / \mathrm{ml}$ prepared in $10 \% \mathrm{MeOH}$ with $0.1 \%$ TFA) were sprayed onto the tissue sections. The investigation used $5 \mu \mathrm{g} / \mathrm{ml}$ of probe, so the incubation time was $15 \mathrm{~min}$ at room temperature. Then three layers of CHCA $(10 \mathrm{mg} / \mathrm{ml}$ prepared in $80 \% \mathrm{ACN}$ ) were sprayed onto the tissue sections after incubation.

MALDI analysis was carried out by Bruker ultrafleXtreme MALDI TOF/TOF (Bruker Daltonik, Bremen, Germany) with 


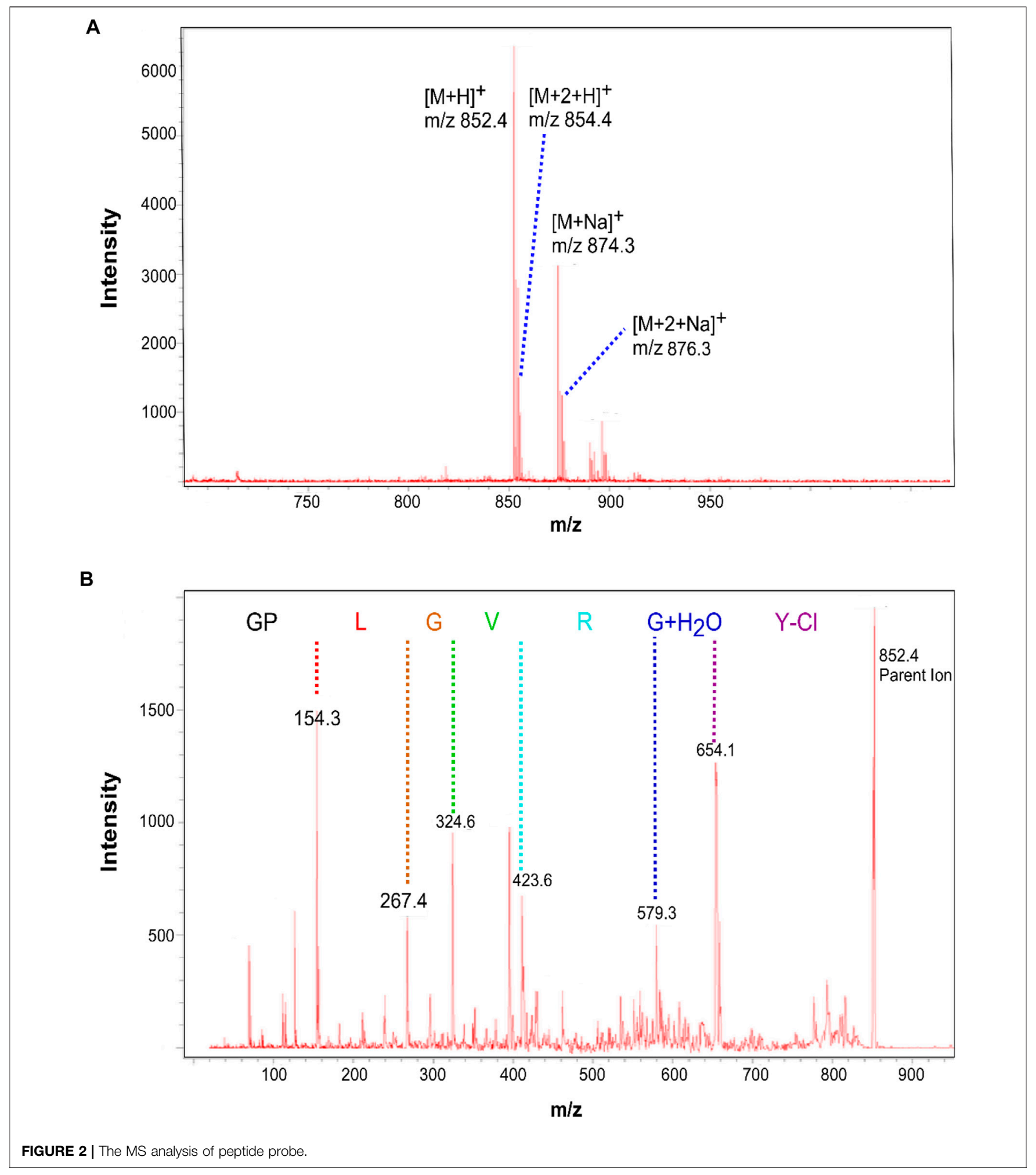

a positive ion mode (delay: $150 \mathrm{~ns}$; ion source one voltage: $20 \mathrm{kV}$; ion source two voltage: $18 \mathrm{kV}$; lens voltage: $6 \mathrm{kV}$ ). All spectra were shown baseline-subtracted, smoothed, and autoscaled in the $Y$-direction, covering a range of $300-3,000 \mathrm{Da}$, with $X$-axis scale increments of $1 \mathrm{Da}$.

\section{RESULTS}

The whole strategy of this method was shown in Figure 1. In our analysis strategy, a peptide probe was synthesized as the substrate of MMP2. For specific identification of this probe in MALDI mass 

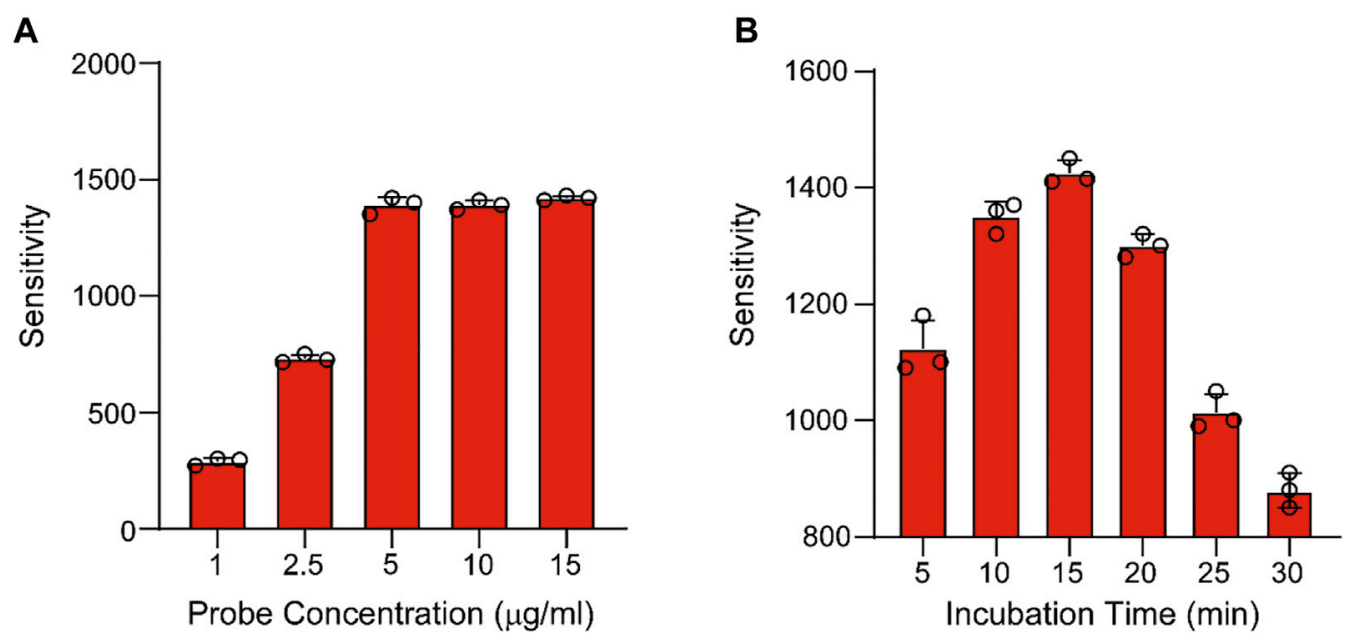

FIGURE 3 | The optimization of the amount (A) and incubation time (B) of probe.

TABLE 1 | clinicopathological features of patients with CRC included in this study.

\begin{tabular}{lc} 
Parameters & $\mathbf{n}=\mathbf{2 0}$ \\
\hline Age (median, range, years) & $62(28-74)$ \\
Gender & $12(60 \%)$ \\
Male & $8(40 \%)$ \\
Female & \\
Infiltration depth & $6(30 \%)$ \\
T1 + T2 & $14(70 \%)$ \\
T3 + T4 & \\
Lymph node metastasis & $13(65 \%)$ \\
Yes & $7(35 \%)$ \\
No & \\
Distant metastasis & $4(20 \%)$ \\
Yes & $16(80 \%)$ \\
No & \\
AJCC TNM Stage & $6(30 \%)$ \\
I + II & $14(70 \%)$ \\
III + IV &
\end{tabular}

spectrometry, $\mathrm{Cl}$ was used binding to the tyrosine terminal, which provided a $[\mathrm{M}+2]^{+}$peak with abundance of $30 \%$. Then the probe can be digested by MMP2 in the tissues and a couple of peaks were detected in MALDI MS $(\mathrm{m} / z 534$ and 536 for enzymedigested product, $m / z 852$ and 854 for peptide probe). Moreover, the ratio between 534 and 852 reflect the amount of peptide probe before and after enzymatic hydrolysis, which can further show the degree of enzymatic hydrolysis, indicating the concentration of MMP2. Thus, this ratio can be considered as the potential biomarker for the quantitative determination of MMP2.

In the experiment, the peptide probe was firstly identified by MALDI MS. From the result (Figure 2), $[\mathrm{M}+\mathrm{H}]^{+}(\mathrm{m} / z$ 852) and $[\mathrm{M}+\mathrm{Na}]^{+}(m / z 874)$ peaks were found with the isotope peaks $[\mathrm{M}+2+\mathrm{H}]^{+}\left(m / z\right.$ 854) and $[\mathrm{M}+2+\mathrm{Na}]^{+}(m / z$ 876), which indicated the probe was successfully synthesized. In addition, tandem mass result showed that the sequence accuracy of the peptide probe. Then, the digestion effect was investigated in vitro. The peptide was incubated by MMP2 enzyme and the digestion products were detected by MS. From the results, the probe was successfully digested by MMP2, with two products in sequence of GPL $(m / z 335)$ and GVRGY-Cl $(m / z 534)$.

After in vitro experiment, the peptide probe was applied into the in vivo experiment for colon cancer tissues. The concentration of probe and the incubation time were also optimized beforehand. The peptide probe was dissolved into $10 \% \mathrm{MeOH}$ with $0.1 \%$ TFA and sprinkled onto the tissues. Then the tissues were put into the MALDI MS to detect the sensitivity of product peak. For probe concentration optimization, five types of concentration, including $1,1.5,5,10$, and $15 \mu \mathrm{g} / \mathrm{ml}$ were studied respectively. When the concentration of probe was over $5 \mu \mathrm{g} / \mathrm{ml}$, the sensitivity of the product remained unchanged, indicating that the optimal concentration of probe was $5 \mu \mathrm{g} / \mathrm{ml}$ (Figure 3A). Next, the incubation time was also optimized. In this investigation, six incubation times, containing $5,10,15,20,25$, and 30 min were compared, in which $15 \mathrm{~min}$ achieved the best sensitivity of product peak (Figure 3B). After method optimization, the method validation was also processed by tissue samples. From our results of probe detection in tissues, the LOD was $50 \mathrm{ng} / \mathrm{ml}$ and the LOQ was $150 \mathrm{ng} / \mathrm{ml}$ from tissue samples. The LOD and the LOQ were $10 \mathrm{ng} / \mathrm{ml}$ and $30 \mathrm{ng} / \mathrm{ml}$ from the blank slides. Moreover, the method validation showed the recovery was over $50 \%$. Since we are testing a pair of peaks, there is almost no falsediscovery.

Finally, MMP2 was reported to be over-expression in several solid tumors, including CRC, gastric carcinoma, breast carcinoma, and lung cancer, etc (Fouad et al., 2019; Ramezani et al., 2020). It has also been reported that MMP2 played a crutial role in tumor invasion and metastasis. For the further understanding its mechanism, the position of MMP2 in tumor tissues need to be determined (Zhang et al., 2019a). Thus, 20 sets of tissue samples from colon cancer patients were collected to prove the ability of our methods. As shown in Table 1, the 


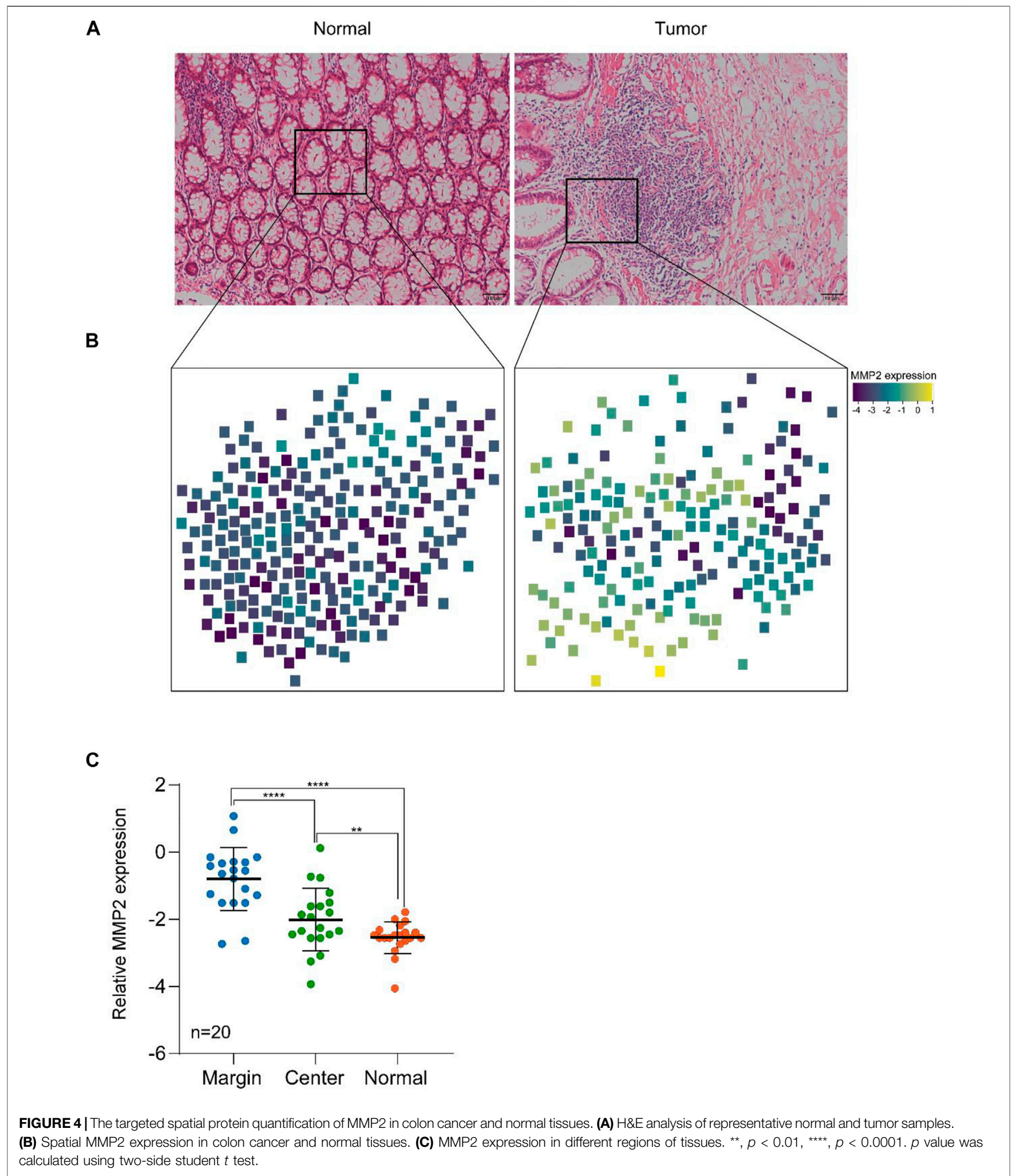

average age of the patients was 62 year-old, and there were 12 men and eight women. Most of the patients were in the deep infiltration depth but some of them had already reached lymph node metastasis or distant metastasis. What we investigated was whether the detected MMP2 expression had a relationship with the tumor invasion. We used a tissue targeted spatial protein quantification strategy based on MALDI TOF analysis for the study of the MMP2 over-expression location sites. In this 
strategy, the peptide probe was firstly sprayed onto the tissues. The optimal amount and the incubation time have investigated above. Then HCCA was used as the matrix and sprayed on the tissues before MALDI analysis. For the detection, 300 points were randomly distributed on the tissue, and the ratio between probe fragments and probe were combined with the coordinates of each point as the indicator for MMP2 expression and location sites. The higher the ratio, the more complete the digestion, and the higher the expression of the MMP2. Finally, the ratio and the coordinates were statistically simulated to achieve the tissue distribution information for the expression of MMP2. In addition, this information will combine with the HE staining to obtain the final expression profile of MMP2.

As shown in Figure 4, the spatial expression of MMP2 in cancer tissue and normal tissue was successfully detected, which having a marked difference. The expression in cancer tissue was significantly higher than that in normal tissue. Additionally, in cancer tissue, the expression of MMP2 was also higher in cancer cells than in normal cells, which was consistent with the literature. Moreover, also in cancer tissues, the expression of MMP2 in marginal sites was significantly higher than that in central sites, indicating that the expression of MMP2 may be related to the tumor invasion. Finally, we counted the expression of MMP2 in 20 pairs tissues of colon cancer patients. The statistical results showed that the expression of MMP2 was higher in the marginal region than in the central region, which was consistent in all cancer tissues.

\section{DISCUSSION}

The infiltration and invasion of CRC had a complex mechanism, which involved multiple steps regulated by gene mutation, RNA expression, and protein modifications. Specially for tumor invasion, the cancer cells did not penetrate the basement membrane (BM) without the help of matrix metalloproteinases (MMPs) (Xu et al., 2019). Therefore, the MMPs can be considered as the important biomarkers to evaluate the tumor invasion (Ivancic et al., 2020, Koga et al., 2019, Kolenčík et al., 2020). MMPs are a series of zinccontaining endopeptidases, which mainly composed of MMP2 and MMP9 (Ceballos et al., 2019). They played a significant role in the degradation of extra-cellular matrix components (Wang et al., 2019; Zhou et al., 2019). Moreover, MMP2 was reported to be over-expressed in several solid tumors, including CRC, gastric carcinoma, breast carcinoma, lung cancer, etc (Fouad et al., 2019; Ramezani et al., 2020). It has also been reported that MMP2 played a crutial role in tumor invasion and metastasis. For the further understanding its mechanism, the position of MMP2 in tumor tissues need to be determined (Zhang et al., 2019b). In addition, if the high expression sites of MMP2 can be quickly detected, it can be applied in the rapid clinical pathology, providing guidance for clinical surgery.

In this study, we developed a strategy for the quantification of MMP2 at the spatial level via the MALDI method by using a peptide probe. Numerous differences were observed in different tissue regions, and the results showed that MMP2 was highly expressed in tumor margins compared to the central region. This clearly show the tumor invasiveness in a quantitative manner, which could be used as an important reference for tumor dissection surgery. It is worth noting that the intensity ratio between the cleaved product and the intact probe may not accurately reflect the ratio, since the efficiency of ionization of the two analytes is unknown. Therefore, this ratio should only be used with caution.

Furthermore, the probes coupled with the MALDI-TOF/MS method can be also used to quantify other proteins, such as the MMP family, the kinase family, and so on. Quantification of these proteins at spatial level is crucial for personalized medicine for tumors and other diseases. MMPs also play important roles in tissue remodeling, which means this method can also benefit patient with serious injuries.

\section{CONCLUSION}

In this work, we have developed a strategy for the MMP2 MALDI spatial protein quantification determination by using a peptide probe, and this method has been successfully applied into the detection of the expression and location sites of MMP2 in colon cancer and normal tissue. In addition, the expression of MMP2 was found to be higher in the margins of cancer tissues, which may be related to the tumor infiltration. This method could help the clinical evaluation of the prognosis for colon cancer patients.

\section{DATA AVAILABILITY STATEMENT}

The raw data supporting the conclusions of this article will be made available by the authors, without undue reservation.

\section{ETHICS STATEMENT}

The studies involving human participants were reviewed and approved by Second Affiliated Hospital of Chengdu Medical College. The patients/participants provided their written informed consent to participate in this study.

\section{AUTHOR CONTRIBUTIONS}

DY, PL, TY, KF, and SZ performed the experiments and participated in article writing. PL, TY, and LC collected the clinical samples. All authors contributed to the article and approved the submitted version.

\section{FUNDING}

This work is supported by the Natural Science Foundation of Sichuan Province (2020YJ0194), the Innovation Project of Chengdu (2021-YF05-01603-SN) and the Young Talent Program of the China National Nuclear Corporation. 


\section{REFERENCES}

Balluff, B., Hopf, C., Porta Siegel, T., Grabsch, H. I., and Heeren, R. M. A. (2021). Batch Effects in MALDI Mass Spectrometry Imaging. J. Am. Soc. Mass. Spectrom. 32, 628-635. doi:10.1021/jasms.0c00393

Barré, F. P. Y., Paine, M. R. L., Flinders, B., Trevitt, A. J., Kelly, P. D., Ait-Belkacem, R., et al. (2019). Enhanced Sensitivity Using MALDI Imaging Coupled with Laser Postionization (MALDI-2) for Pharmaceutical Research. Anal. Chem. 91, 10840-10848. doi:10.1021/acs.analchem.9b02495

Boskamp, M. S., and Soltwisch, J. (2020). Charge Distribution between Different Classes of Glycerophospolipids in MALDI-MS Imaging. Anal. Chem. 92, 5222-5230. doi:10.1021/acs.analchem.9b05761

Ceballos, M. P., Rigalli, J. P., Ceré, L. I., Semeniuk, M., Catania, V. A., and Ruiz, M. L. (2019). ABC Transporters: Regulation and Association with Multidrug Resistance in Hepatocellular Carcinoma and Colorectal Carcinoma. Curr. Med. Chem. 26, 1224-1250. doi:10.2174/0929867325666180105103637

Fouad, H., Salem, H., Ellakwa, D. E. S., and Abdel-Hamid, M. (2019). MMP-2 and MMP-9 as Prognostic Markers for the Early Detection of Urinary Bladder Cancer. J. Biochem. Mol. Toxicol. 33, e22275. doi:10.1002/jbt.22275

Griselda, A., Idalia, G., Claudia, C., and Jesús, R. (2020). The Roles of Matrix Metalloproteinases and Their Inhibitors in Human Diseases. Int. J. Mol. Sci. 21 (24), 9739. doi:10.3390/ijms21249739

Ivancic, M. M., Megna, B. W., Sverchkov, Y., Craven, M., Reichelderfer, M., Pickhardt, P. J., et al. (2020). Noninvasive Detection of Colorectal Carcinomas Using Serum Protein Biomarkers. J. Surg. Res. 246, 160-169. doi:10.1016/j.jss.2019.08.004

Koga, Y., Hirahashi, M., Ohishi, Y., and Oda, Y. (2019). Clinicopathological Features and Phenotypic Classification of De Novo-Type Colorectal Carcinomas Differ from Those of Colorectal Carcinomas Derived from Flat Adenomas. Pathol. Int. 69, 331-340. doi:10.1111/pin.12803

Kolenčík, D., Shishido, S. N., Pitule, P., Mason, J., Hicks, J., and Kuhn, P. (2020). Liquid Biopsy in Colorectal Carcinoma: Clinical Applications and Challenges. Cancers 12, 1376. doi:10.3390/cancers12061376

Montalban-Arques, A., and Scharl, M. (2019). Intestinal Microbiota and Colorectal Carcinoma: Implications for Pathogenesis, Diagnosis, and Therapy. EBioMedicine 48, 648-655. doi:10.1016/j.ebiom.2019.09.050

Moore, L. M., Cho, S., and Thoren, K. L. (2019). MALDI-TOF Mass Spectrometry Distinguishes Daratumumab from M-Proteins. Clinica Chim. Acta 492, 91-94. doi:10.1016/j.cca.2019.02.017

Ramezani, P., Abnous, K., Taghdisi, S. M., Zahiri, M., Ramezani, M., and Alibolandi, M. (2020). Targeted MMP-2 Responsive Chimeric Polymersomes for Therapy against Colorectal Cancer. Colloids Surf. B: Biointerfaces 193, 111135. doi:10.1016/j.colsurfb.2020.111135

Ryan, D. J., Spraggins, J. M., and Caprioli, R. M. (2019). Protein Identification Strategies in MALDI Imaging Mass Spectrometry: A Brief Review. Curr. Opin. Chem. Biol. 48, 64-72. doi:10.1016/j.cbpa.2018.10.023
Shariatgorji, M., Nilsson, A., Fridjonsdottir, E., Vallianatou, T., Källback, P., Katan, L., et al. (2019). Comprehensive Mapping of Neurotransmitter Networks by MALDI-MS Imaging. Nat. Methods 16, 1021-1028. doi:10.1038/s41592-0190551-3

Wang, Z., Wang, Y., Jia, X., Han, Q., Qian, Y., Li, Q., et al. (2019). MMP-2Controlled Transforming Micelles for Heterogeneic Targeting and Programmable Cancer Therapy. Theranostics 9, 1728-1740. doi:10.7150/ thno.30915

Weng, X., Chen, W., Hu, W., Xu, K., Qi, L., Chen, J., et al. (2019). PTPRB Promotes Metastasis of Colorectal Carcinoma via Inducing Epithelial-Mesenchymal Transition. Cell Death Dis 10, 352. doi:10.1038/s41419-019-1554-9

Xu, Y., Zhang, J., Liu, X., Huo, P., Zhang, Y., Chen, H., et al. (2019). MMP-2Responsive Gelatin Nanoparticles for Synergistic Tumor Therapy. Pharm. Develop. Technol. 24, 1002-1013. doi:10.1080/10837450.2019.1621899

Zhang, D., Yang, Y., Qin, Q., Xu, J., Wang, B., Chen, J., et al. (2019). MALDI-TOF Characterization of Protein Expression Mutation during Morphological Changes of Bacteria under the Impact of Antibiotics. Anal. Chem. 91, 2352-2359. doi:10.1021/acs.analchem.8b05080

Zhang, H., Lu, Y., Wu, J., and Feng, J. (2019a). LINC00460 Hypomethylation Promotes Metastasis in Colorectal Carcinoma. Front. Genet. 10, 880. doi:10.3389/fgene.2019.00880

Zhang, H., Ma, Y., Wang, H., Xu, L., and Yu, Y. (2019b). MMP-2 Expression and Correlation with Pathology and MRI of Glioma. Oncol. Lett. 17, 1826-1832. doi:10.3892/ol.2018.9806

Zhou, W., Yu, X., Sun, S., Zhang, X., Yang, W., Zhang, J., et al. (2019). Increased Expression of MMP-2 and MMP-9 Indicates Poor Prognosis in Glioma Recurrence. Biomed. Pharmacother. 118, 109369. doi:10.1016/ j.biopha.2019.109369

Conflict of Interest: The authors declare that the research was conducted in the absence of any commercial or financial relationships that could be construed as a potential conflict of interest.

Publisher's Note: All claims expressed in this article are solely those of the authors and do not necessarily represent those of their affiliated organizations, or those of the publisher, the editors, and the reviewers. Any product that may be evaluated in this article, or claim that may be made by its manufacturer, is not guaranteed or endorsed by the publisher.

Copyright $\odot 2021$ Yu, Lai, Yan, Fang, Chen and Zhang. This is an open-access article distributed under the terms of the Creative Commons Attribution License (CC BY). The use, distribution or reproduction in other forums is permitted, provided the original author(s) and the copyright owner(s) are credited and that the original publication in this journal is cited, in accordance with accepted academic practice. No use, distribution or reproduction is permitted which does not comply with these terms. 